\title{
Aplicaciones de los Métodos Computacionales al Estudio de la Estructura y Propiedades de Polímeros
}

\author{
Carlos Alemán, Sebastián Muñoz-Guerra \\ Departament d’Enginyeria Química, Universitat Politécnica de Catalunya, España
}

\begin{abstract}
Resumo: En este trabajo se revisan las técnicas de simulación molecular más habituales y potentes para la descripción de los polímeros a escala atómica y molecular, las cuales se han clasificado en cuánticas o clásicas dependiendo de cómo se describen las interacciones entre las partículas. Se presentan asimismo diversas aplicaciones de dichas metodologías, realizadas en nuestro laboratorio, en el contexto del estudio de la estructura y propiedades de polímeros. En particular, se muestran aplicaciones de las técnicas clásicas a la determinación de estructuras cristalinas, a estudio del plegamiento lamelar de los nylons, a la estabilidad de las estructuras supramoleculares observadas en algunos complejos tensioactivo polielectrolito y a la difusión de gases en matrices poliméricas, mientras que el uso de técnicas cuánticas se ha ilustrado presentando estudios dedicados a la predicción de efectos cooperativos, interacciones específicas y parámetros espectroscópicos.
\end{abstract}

Palabras-claves: Métodos computacionales, simulación molecular, polímeros, estudio teórico.

\section{On the Applicability of Computational Methods to Study the Structure and Properties of Polymers}

Abstract: The most important computational techniques employed to simulate the structure and properties of polymers at the miscroscopic level have been reviewed. They have been classified in quantum or classical methods depending on the expressions used to describe the interactions between the particles. Furthermore, the applicability of such modeling tools is shown with some results recently obtained by our group. More specifically, we show the applicability of classical methods to determine the crystal structure of polymers, to model the lamellar folding of nylons, to predict the stability of the supramolecular structures adopted by some surfactant-polyelectrolite self-assembled complexes and to investigate the diffusion of simple gases through polymeric matrices. The reliability of quantum methods has been illustrated displaying some studies devoted to study cooperative effects, specific interactions and spectroscopic parameters.

Keywords: Computational methods, molecular simulation, polymers, theoretical study, classical mechanics and quantum mechanics

\section{Introducción}

La aparición de ordenadores en el mundo de la química es relativamente reciente. Sin embargo, debido al rápido aumento de su potencia, se han convertido en una herramienta de gran valor para el estudio y comprensión de los sistemas químicos. Su uso abarca desde la creación y administración de bases de datos a la simulación computacional a escala atómica de procesos químicos y físicos. De hecho, a lo largo de las últimas dos décadas la química computacional ha evolucionado hasta convertirse en una potente herramienta que no solo permite a los investigadores racionalizar los resultados extraídos de la experimentación, sino también obtener un tipo de información absolutamente inasequible para las metodologías experimentales. La calidad de los resultados obtenidos ha permitido que las técnicas computacionales se hayan generalizado a diversos campos de la Química y la Biología, por ejemplo la Química Orgánica e Inorgánica, la Farmacología, o la Biología Molecular. Recientemente ha sido la Ciencia de los Polímeros la que de forma decidida ha incorporado su uso al estudio de los polímeros sintéticos.

Las propiedades de cualquier material polimérico están directa o indirectamente ligadas a su estructura molecular. Es decir, son una consecuencia de la estructuración de las macromoléculas que constituyen un determinado material $\mathrm{y}$, en muchos casos, son un reflejo de los procesos dinámicos que se dan a escala molecular. Entender y racionalizar la estructura de un material debe permitir comprender el origen de sus propiedades físicas. De ahí que el estudio de la estructura molecular de los materiales poliméricos -escala microscópica- y su relación con las propiedades observables -escala macroscópica- se haya convertido en uno de los campos de investigación que más interés despierta. En este sentido resulta evidente la importancia de abordar estos estudios mediante técnicas computacionales basadas en la simulación molecular, ya que estas permitirán obtener detalles a nivel atómico que son difíciles cuando no imposibles de investigar desde un punto de vista experimental.

Autor para correspondência: Carlos Alemán, Departament d’Enginyeria Química, Universitat Politécnica de Catalunya, Diagonal 647, Barcelona E-08028, España. E-mail: carlos.aleman@upc.es 
La incorporación del ordenador al estudio de los materiales poliméricos es reciente, y para ello fue preciso el desarrollo de métodos teóricos capaces de representar sistemas químicos de naturaleza macromolecular. Como fruto de tales trabajos, disponemos actualmente de un gran número de metodologías capaces de reproducir y predecir propiedades de los polímeros únicamente a partir de su estructura molecular. Estos métodos proporcionan una visión muy detallada de los sistemas poliméricos, por lo que es posible obtener información microscópica sobre las interacciones moleculares, que no es accesible a las técnicas macroscópicas. En este sentido, las técnicas de simulación molecular constituyen en la actualidad unas herramientas muy potentes para estudiar el origen microscópico y molecular de diferentes fenomenologías observadas experimentalmente.

El objetivo de este artículo divulgativo es aproximar al lector a este campo de investigación. En primer lugar, se presentarán las técnicas de simulación más potentes para la descripción microscópica de sistemas químicos, en general, y macromoleculares, en particular. A continuación se revisarán algunas de sus posibles aplicaciones en el contexto de la Ciencia de los Polímeros, que ilustraremos en base a la experiencia adquirida por nuestro grupo de investigación durante los últimos años.

\section{Técnicas de simulación molecular}

Los métodos de simulación molecular que permiten obtener información microscópica del sistema objeto de estudio se pueden agrupar en dos grandes grupos: los métodos cuánticos y los métodos clásicos. Los primeros representan el sistema molecular mediante un conjunto de núcleos y electrones, que siguen las leyes fundamentales de la Mecánica Cuántica. Los métodos clásicos describen el sistema como un conjunto de partículas elementales, localizadas sobre los núcleos, y cuyas interacciones se aproximan a una suma de términos energéticos representados por expresiones basadas en la Mecánica Clásica. El uso de potenciales clásicos implica una descripción menos rigurosa del sistema, pero abarata en gran medida los gastos computacionales.

\section{Técnicas cuánticas}

Las técnicas computacionales que ofrecen más confianza y rigurosidad son las basadas en la Mecánica Cuántica. Estos métodos permiten obtener de forma fiable propiedades tales como la geometría o la energía de sistemas químicos, y dado que consideran los electrones explícitamente, pueden cuantificar las propiedades relacionadas con estos (parámetros espectroscópicos, distribución de cargas, momento dipolar, etc.). Sin embargo, estas ventajas se obtienen a cambio de unos requerimientos computacionales muy elevados y por lo tanto, solo se pueden aplicar a sistemas constituidos por pocos átomos.

La Mecánica Cuántica postula la existencia de una función de onda (o función de estado), simbolizada por $\psi$, que contiene toda la información posible del sistema. Para obte- ner esta función es necesario resolver la celebre ecuación de Schrödinger:

$$
\mathrm{H} \psi=\mathrm{E} \psi
$$

donde $H$ es el operador hamiltoniano que incluye la energía cinética y potencial de núcleos y electrones, y $E$ la energía del sistema. A pesar de su aparente simplicidad, la resolución analítica de esta ecuación solo es posible para sistemas de tipo hidrogenoide. Con el objetivo de estudiar sistemas de mayor tamaño se han de introducir toda una serie de simplificaciones entre las que destaca la aproximación de BornOppenheimer, la cual considera, de forma muy razonable, que el movimiento de los núcleos es mucho más lento que el de los electrones. Otras simplificaciones que se introducen son la representación de la función de onda en términos de orbitales moleculares (aproximación orbital), y la expresión de estos orbitales como combinación lineal de orbitales atómicos (aproximación MO-LCAO).

En la práctica existen tres metodologías básicas para la obtención de la función de onda: la ab initio, la semiempírica y la basada en la Teoría del Funcional de la Densidad (DFT). La filosofía de las tres metodologías es completamente diferente. Los métodos $a b$ initio ${ }^{[1]}$ emplean el formalismo químico cuántico de manera rigurosa, no empleando parámetros empíricos más allá de las magnitudes físicas más elementales. El método del campo autoconsistente o de Hartree-Fock (HF), que es el más sencillo entre los ab initio, se basa en el teorema variacional y pretende encontrar la función de onda que minimiza la energía del sistema. La función de onda se calcula considerando que cada electrón se mueve en un campo debido a los núcleos y un campo promedio creado por todos los demás electrones. Así, el método HF no incluye la interacción entre cada electrón y el resto de los electrones individualmente (correlación electrónica) lo que provoca una sobre-estimación de la repulsión electrónica.

La manera más sencilla de incluir la correlación electrónica es la interacción de configuraciones (CI). Para ello se representa el estado electrónico fundamental de la molécula por una mezcla de todos los estados electrónicos posibles (cálculos full-CI). Sin embargo, muchas veces la expansión debe ser truncada a solo unos pocos estados electrónicos accesibles (cálculos truncated-CI). En general, la CI permite obtener la descripción no relativista más completa posible de un sistema molecular. No obstante, este método es resulta prohibitivo para sistemas de tamaño medio. Otra manera de tratar la correlación electrónica son los métodos perturbacionales. Sin duda, el más empleado es el de MøllerPlesset (MP), el cual se basa en la teoría de de Rayleigh y Schrödinger.

Los métodos semiempíricos nacieron con el propósito de proporcionar unas técnicas mecanocuánticas para el estudio de propiedades moleculares, de forma que siendo suficientemente precisos y fiables como para tener un valor práctico en la investigación química, no presenten los inconvenientes de los procedimientos $a b$ initio, esto es, que sean aplicables a grandes moléculas. Para ello se introducen una serie de sim- 
plificaciones en la expresión del hamiltoniano HF, que se compensan con la inclusión de un conjunto de parámetros. Entre los métodos semiempíricos más populares en la actualidad cabría destacar el Austin Model 1 (AM1) ${ }^{[2]}$ y el Parametrizatión Method 3 (PM3) ${ }^{[3]}$ desarrollados por Dewar y Stewart, respectivamente.

La aproximación $\mathrm{DFT}^{[4]}$ se basa en los teoremas de Hohenberg-Kohn que demuestran la existencia de un funcional de la densidad electrónica que determina la energía del estado fundamental, si bien, no determinan la forma del funcional. Además, la densidad electrónica puede ser representada como la suma de $N$ densidades de orbitales monoelectrónicos, lo que hace que los cálculos DFT puedan ser fácilmente aplicables. A partir de estas ideas se calcula energía electrónica como la suma de varios términos que son función de la densidad electrónica, $\rho$. Los método DFT se han empezado a aplicar a sistemas de interés químico hace relativamente poco tiempo. Sin embargo, la calidad de los resultados obtenidos, así como su eficiencia computacional, hacen que en la actualidad se empleen cada vez con más frecuencia, reemplazando en muchas ocasiones a los cálculos CI y MP.

\section{Técnicas clásicas}

En los métodos clásicos, la energía de un sistema se calcula sumando la contribución asociada a los átomos que están enlazados entre si $\left(\mathrm{E}_{\text {enlazante }}\right)$ a la contribución que contabiliza la interacciones entre átomos no enlazados $\left(\mathrm{E}_{\text {no-enlazante }}\right)^{[5,6]}$ :

$$
\mathrm{E}=\mathrm{E}_{\text {enlazante }}+\mathrm{E}_{\text {no-enlazante }}
$$

El término enlazante a su vez se suele determinar como suma de tres términos energéticos: stretching $\left(\mathrm{E}_{\mathrm{str}}\right)$, bending $\left(\mathrm{E}_{\mathrm{bnd}}\right)$ y torsión $\left(\mathrm{E}_{\mathrm{tor}}\right)$. El stretching y el bending están relacionados con la distorsión de las distancias (I) y ángulos $(\theta)$ de enlace, respectivamente, y se suelen expresar mediante funciones de tipo armónico. Por otro lado el término de torsión describe las barreras de rotación respecto a los enlaces químicos, las cuales se describen mediante ángulos diedros $(\varphi)$. La energía de torsión se calcula a partir de sumas de Fourier que vienen definidas por tres parámetros: el diedro más estable $(\gamma)$, la periodicidad $(n)$ y la altura de la barrera $\left(\mathrm{V}_{\mathrm{n}}\right)$.

$$
\begin{aligned}
E_{\text {enlazante }} & =E_{\text {str }}+E_{\text {bnd }}+E_{\text {tor }}= \\
& =k_{s t r}\left(1-1_{0}\right)^{2}+k_{\text {bnd }}\left(\theta-\theta_{0}\right)^{2}+\Sigma V_{n} / 2[1+\cos (n \varphi-\gamma)]
\end{aligned}
$$

El término no-enlazante es el encargado de representar las interacciones electrostáticas y de van der Waals de las moléculas. Estas interacciones se calculan en principio para todos los pares de átomos no unidos. La fórmula más usual para expresar la energía de van der Waals $\left(\mathrm{E}_{\mathrm{vdW}}\right)$ es la denominada potencial de Lennard-Jones 12-6. El término repulsivo, con signo positivo, se representa como $\mathrm{r}^{-12}$, mientras que para el término atractivo, con signo negativo, se emplea una función $\mathrm{r}^{-6}$. La energía electrostática $\left(\mathrm{E}_{\mathrm{ele}}\right)$ se suele calcular mediante una simple expresión de tipo Coulómbico haciendo uso de cargas localizadas sobre los átomos.

$$
\mathrm{E}_{\text {no-enlazante }}=\mathrm{E}_{\mathrm{vdW}}+\mathrm{E}_{\text {ele }}=\varepsilon^{*}\left[\left(\mathrm{r}^{*} / \mathrm{r}\right)^{12}-2\left(\mathrm{r}^{*} / \mathrm{r}\right)^{6}\right]+\mathrm{q}_{\mathrm{i}} \mathrm{q}_{\mathrm{j}} / \mathrm{r}
$$

En estas fórmulas, $r$ es la distancia entre los átomos, $r^{*}$ está relacionado con los radios de van der Waals de los átomos implicados, $\varepsilon^{*}$ con los parámetros de dureza de cada átomo, $\mathrm{y} \mathrm{q}_{\mathrm{i}} \mathrm{y} \mathrm{q}_{\mathrm{j}}$ son las cargas atómicas.

El cálculo de la energía a partir de técnicas clásicas se puede aplicar a diferentes algoritmos con el objetivo de realizar simulaciones que permitan describir las propiedades del sistema. Estos algoritmos son fundamentalmente tres: Minimimización, Dinámica Molecular y Monte Carlo ${ }^{[7]}$. Como su nombre indica, el primero de ellos consiste en un algoritmo de minimización para optimizar la energía del sistema y caracterizar los puntos singulares obtenidos. La Minimización es sin duda el más simple de los métodos basados en técnicas clásicas, sin embargo hay que enfatizar que puede emplearse en multitud de aplicaciones, como por ejemplo, el estudio estructural de macromoléculas en fases condensadas, análisis conformacional, estudio de frecuencias de vibración, evaluación de propiedades termodinámicas y mecánicas, etc.

La Dinámica Molecular es un algoritmo que intenta simular el comportamiento de un sistema sometiendo sus átomos a las leyes de la Mecánica Newtoniana. La trayectoria de la simulación se efectúa integrando las ecuaciones de Newton del movimiento a lo largo del tiempo para todas las partículas del sistema.

$$
\mathrm{F}_{i}(\mathrm{t})=\mathrm{m}_{\mathrm{i}} \mathrm{a}_{\mathrm{i}}(\mathrm{t})=\mathrm{m}_{\mathrm{i}} \mathrm{d}^{2} \mathrm{r}_{\mathrm{i}}(\mathrm{t}) / \mathrm{dt}^{2}
$$

donde $F_{i}(t), a_{i}(t)$ y $r_{i}(t)$ son, respectivamente, la fuerza sobre el átomo $i$, su aceleración y su posición el tiempo. La fuerza se calcula como la primera derivada de la energía potencial:

$$
\mathrm{Fi}(\mathrm{t})=-\partial \mathrm{V}(\mathrm{r} 1, \mathrm{r} 2, \ldots . \mathrm{rN}) / \partial \mathrm{ri}
$$

Una vez se ha obtenido la fuerza que ejerce el sistema sobre una de las partículas, se divide por su masa y así se calcula la aceleración que experimenta esa partícula. A partir de la posición y velocidad actuales de las partículas del sistema y conociendo la aceleración es posible calcular nuevas posiciones y velocidades un instante de tiempo después mediante un algoritmo de integración. De esta forma se obtiene la trayectoria que describe el sistema a lo largo del tiempo.

La técnica de Monte Carlo, es una metodología estocástica en la que los hechos estructurales y las propiedades termodinámicas se obtienen a partir de promedios. Así, el método de Monte Carlo intenta obtener un muestreo de Boltzmann de un sistema según un algoritmo que consiste en generar estructuras al azar, evaluar su energía y decidir a partir de un criterio adecuado si la nueva estructura generada se acepta o se descarta. El formalismo de Monte Carlo más simple es el algoritmo de Metropolis ${ }^{[7]}$, en el que la generación de nuevas estructuras tiene lugar de forma aleatoria, asegurándose la reversibilidad microscópica del sistema. Sin embargo, en sistemas químico muy densos, como son los polímeros, el método de Metropolis resulta poco eficiente y es preciso emplear algoritmos mas sofisticados. En este sentido el algoritmo denominado Configurational 
Bias, el cual se basa en generar movimientos por reconstrucción para obtener nuevas estructuras, ha resultado tremendamente útil para estudiar sistemas poliméricos tanto en dominios amorfos ${ }^{[8]}$ como en dominios cristalinos $^{[9]}$.

\section{Aplicaciones de las técnicas de simulación molecular}

El abanico de problemas abordables por las técnicas de simulación molecular ha experimentado un gran expansión durante la última década. Debido a las características estructurales de los materiales poliméricos, los estudios basados en el uso de técnicas de simulación molecular pueden centrarse en uno de sus dos tipos de organización: la fase amorfa y la fase cristalina. La descripción de la fase amorfa mediante metodologías teóricas resulta especialmente compleja debido a la falta de regularidad conformacional, al grado más o menos elevado de movimientos moleculares y el número elevado de átomos explícitos que es necesario considerar para obtener una representación adecuada del sistema. No obstante, en las últimas décadas se han desarrollado estrategias computacionales, en algunos casos relativamente sofisticadas, que combinadas de forma apropiada con los métodos de simulación presentados en la sección anterior, son capaces de proporcionar una descripción precisa de los polímeros amorfos $^{[10-19]}$. Sin embargo, la exposición de estrategias teóricas específicas está fuera de los objetivos de este trabajo de revisión. En consecuencia, hemos decidido centrarnos en presentar la aplicación de técnicas de simulación molecular a la fase cristalina, ya que no se precisa de metodologías específicas adicionales. Concretamente, en esta sección se mostrarán algunas de las aplicaciones que han formado parte de nuestra investigación durante los últimos años.

\section{Determinación de la estructura cristalina de poliamidas no convencionales}

La determinación de la estructura cristalina de un polímero no es una cuestión simple. Así, la calidad y cantidad de información experimental que se obtiene por difracción de rayos $\mathrm{X}$ está limitada por las propiedades inherentes a la muestra utilizada: tamaño del cristal, defectos estructurales, falta de orientación de los especímenes, etc. En consecuencia, es muy frecuente que la información que proporcionan las técnicas de difracción resulte insuficiente para determinar la estructura de un polímero a nivel atómico ${ }^{[20]}$. Por este motivo, las técnicas de simulación molecular se han convertido en un complemento, y en algunos casos una alternativa, a las técnicas tradicionalmente empleadas para estudios estructurales $^{[20-23]}$. En concreto, las herramientas computacionales no sólo se emplean para resolver la estructura cristalina de un polímero a partir de una información experimental limitada, sino que además permiten comprender los factores físicoquímicos que determinan su estructuración ${ }^{[24]}$.

Los métodos basados en el acoplamiento de un algoritmo de minimización con el cálculo de la energía a partir de potenciales clásicos, combinados adecuadamente con la información experimental disponible (Figura 1), se emplean

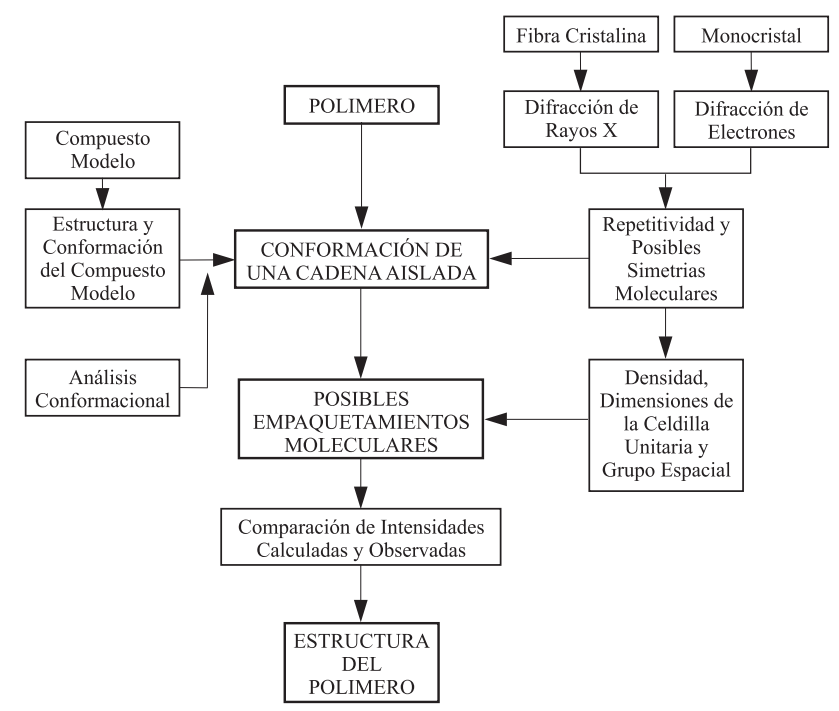

Figura 1. Diagrama de flujo del procedimiento seguido para establecer la estructura cristalina de un polímero a partir de los datos de difracción de rayos $\mathrm{X}$ y electrones combinados con el uso de metodologías computacionales.

habitualmente para determinar a nivel atómico la estructuras cristalina de los polímeros ${ }^{[20,25,26]}$. Entre los polímeros estudiados en nuestro laboratorio destacan los poli $(\alpha$-alquil- $\beta, \mathrm{L}-$ aspartato)s, abreviados PAALAs, que pueden considerarse como derivados del nylon 3 sustituidos estereorregularmente.

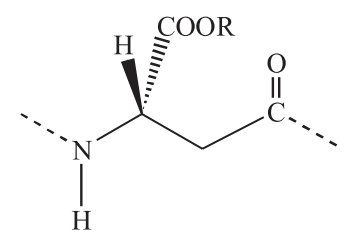

PAALAs: poli( $\alpha$-alquil- $\beta$, L-aspartato)

La característica estructural más importante de esta familia de poliamidas es su tendencia a adoptar conformaciones helicoidales similares a las de las proteínas. Sin embargo, los PAALAs presentan una cierta diversidad estructural. Así, la simetría de la conformación helicoidal y el empaquetamiento cristalino varían en función de la longitud y/o la constitución química del grupo alquilo $R$. La combinación de la información obtenida a partir de difracción de rayos $\mathrm{X}$ y electrones, y los resultados conseguidos mediante métodos de Minimización de la energía permitieron describir la estructura cristalina de estos polímeros.

La hélice 13/4 (Figura 2a) se trata sin duda de la conformación más frecuentemente observada para los PAALAs. Así, se encuentra presente en los derivados con grupos alquilo lateral de tamaño mediano, tanto lineales como ramificados ${ }^{[27-30]}$. Esta conformación presenta una simetría de 3.25 residuos por vuelta de hélice y está estabilizada por puentes de hidrógeno intramoleculares entre los grupos amida $i$ e $i+3$. En estado sólido, esta conformación suele presentarse en un empaquetamiento de tipo pseudohexagonal constituidos por dos cadenas con orientación antiparalela dentro de la celda unidad (Figura 3), el cual corresponde al grupo espacial $P 2_{1}$. 

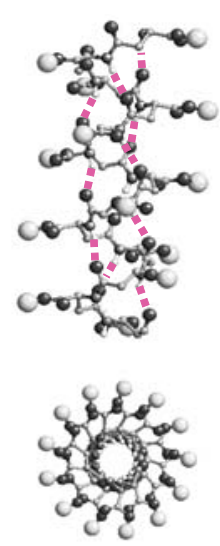

(a)
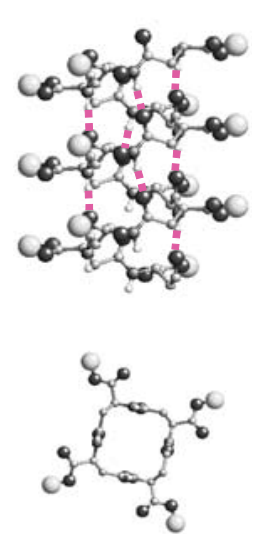

(b)
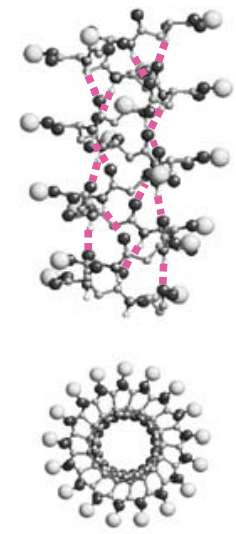

(c)

Figura 2. Proyección axial y ecuatorial de una hélice 13/4 (a), 4/1 (b) y $17 / 4$ (c) para los PAALAs.

La hélice 4/1 (Figure 2b) únicamente se ha observado en polímeros con cadenas laterales ramificadas y lineales de tamaño pequeño o medio ${ }^{[27,28,31]}$. Se trata de una hélice con simetría integral, de 4 residuos por vuelta, estabilizada por puentes de hidrógeno intramoleculares entre los grupos amida $i$ e $i+4$. La estructura cristalina en que se encuentra esta hélice corresponde al grupo espacial $P 4_{1}$ y consiste en un empaquetamiento tetragonal, con una única cadena por celda unitaria (Figura 3).

(a)

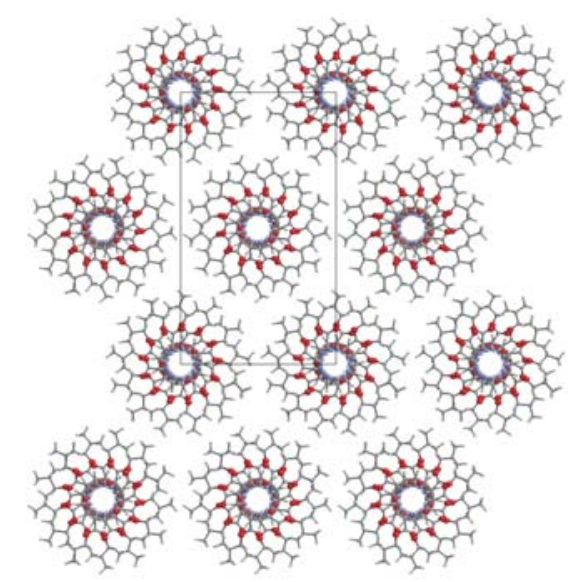

(b)

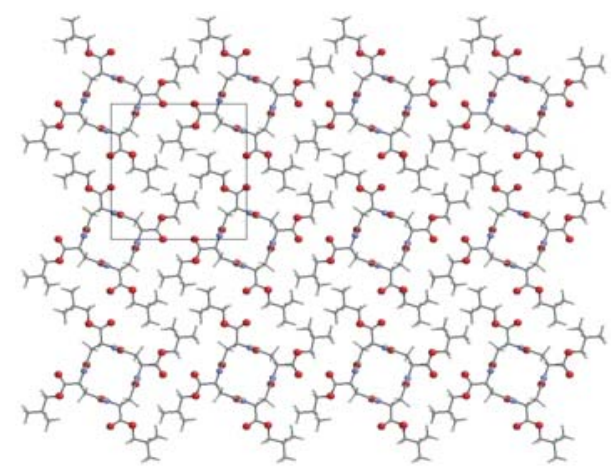

Figura 3. Empaquetamiento de la forma pseudohexagonal (a) y tetragonal (b) del poli $(\alpha$-isobutil- $\beta$-L-aspartato). La celda unitaria está indicada mediante una línea continua. La distancia entre cadenas ha sido aumentada para mayor claridad de las imágenes.

Además de las conformaciones anteriormente descritas, también se han observado en determinados casos las hélices 17/4 y 16/5. La hélice 17/4 ha sido descrita únicamente para los PAALAs con los grupos alquilo más pequeños, es decir los derivados metílico y etílico ${ }^{[28]}$. Conformacionalmente puede ser descrita como un caso intermedio entre las hélices $13 / 4$ y 4/1. Así, su morfología es similar a la de la hélice 13/4, pero los puentes de hidrógeno se forman entre los grupos amida $i \mathrm{e}$ $i+4$ (Figura 2c). La estructura pseudohexagonal en que aparece esta hélice es análoga a la descrita para la hélice 13/4. Por otro lado, en algunos casos los PAALAs con cadenas alquílicas ramificadas adoptan una hélice $16 / 5^{[30]}$. Esta conformación está estrechamente relacionada con la hélice 13/4, presentando idéntico esquema de puentes de hidrógeno y siendo el número de residuos por vuelta muy similar.

\section{Estudio de efectos cooperativos en polímeros y polipéptidos}

Las técnicas cuánticas, al considerar efectos electrónicos, pueden emplearse para evaluar fenómenos cooperativos en la estabilidad intrínseca de una estructura determinada. Para ello es necesario calcular el incremento de energía (IE) en ausencia de efectos cooperativos que se produce cuando se añade un residuo a la cadena polimérica. Habitualmente este valor se obtiene a partir de la diferencia entre la energía de las moléculas constituidas únicamente por uno y dos residuos.

$$
\mathrm{IE}=\mathrm{E}(2)-\mathrm{E}(1)
$$

Así, la energía esperada $\left(\mathrm{E}^{\mathrm{e}}\right)$ para una cadena constituida por $n$ residuos es:

$$
\mathrm{E}^{\mathrm{e}}(n)=(n-1) \mathrm{IE}+\mathrm{E}(1)
$$

La diferencia entre la energía esperada y la energía obtenida a partir de un cálculo mecanocuántico $\left(\mathrm{E}^{\mathrm{QM}}\right)$ corresponde a la energía cooperativa $\left(\mathrm{E}^{\mathrm{coop}}\right)$ para una determinada estructura.

$$
\mathrm{E}^{\mathrm{coop}}=\mathrm{E}^{\mathrm{QM}}-\mathrm{E}^{\mathrm{e}}
$$

La Tabla 1 compara los valores de $\mathrm{E}^{\text {coop }}$ de la hélice $\alpha$ obtenidos a partir de cálculos semiempíricos AM1 para dife-

Tabla 1. Energía Cooperativa ( $\left.\mathrm{E}^{\mathrm{coop}}\right)$ obtenida a partir de cálculos mecanocuánticos para la hélice $\alpha$ en algunos polímeros y polipéptidos.

\begin{tabular}{ccc}
\hline Polipéptido/Polímero & $\mathbf{E}^{\text {coop }}(\mathbf{k c a l} / \mathbf{m o l})^{\mathbf{a}}$ & Referencia \\
\hline Polialanina & -16.7 & {$[25]$} \\
Ácido poli $(\alpha-L-$ glutámico $)$ & -17.1 & {$[26]$} \\
Poli $(\alpha$-metil- $\beta$-L-aspartato $)$ & -19.5 & {$[27]$} \\
Poli $(\alpha$-etil- $\beta$-L-aspartato $)$ & -21.7 & {$[27]$} \\
Poli $(\alpha$-butil- $\beta-L-$ aspartato $)$ & -21.9 & {$[27]$} \\
Poli $(\alpha-$-isobutil- $\beta$-L-aspartato $)$ & -21.1 & {$[27]$} \\
\hline
\end{tabular}

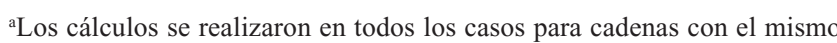
número de residuos. 
rentes polipéptidos y polímeros ${ }^{[32-34]}$. La $\mathrm{E}^{\text {coop }}$ proporciona una medida del grado de estabilización que adquiere dicha conformación cuando el número de residuos en la cadena aumenta. Puede observarse que los PAALAs presentan un fuerte tendencia a adoptar conformaciones helicoidales. Además, esta tendencia parece estar poco influenciada por la naturaleza química (lineal o ramificada) y la longitud de la cadena alquílica lateral. Por otro lado, los valores indicados en la Tabla 1 ponen de manifiesto que la tendencia de los PAALAs a adoptar una conformación helicoidal es incluso superior a la de algunos poli( $\alpha$-péptido)s, como la polialanina o el ácido poli( $\alpha$-glutámico). Esta tendencia también ha sido observada por Seebach y colaboradores para algunos otros poli( $\beta$-péptido $) s^{[35]}$.

Por otro lado, cálculos mecanocuánticos basados en métodos $\mathrm{HF}^{[36-38]}$, $\mathrm{DFT}^{[36]}$ y semiempíricos ${ }^{[32,36,37]}$ han permitido estudiar los efectos cooperativos asociados a la formación de ciertas estructuras secundarias habituales en los polipéptidos. Los resultados pusieron de manifiesto la ausencia de cooperatividad en estructuras semi-extendidas tales como la hoja $\beta$ y la hélice $2_{7}^{[36,37]}$, mientras que la formación de conformaciones helicoidales, muy en particular de la hélice $\alpha$, está potenciada por la presencia de notables fenómenos cooperativos ${ }^{[32,36-38]}$. Además, los resultados permitieron constatar que la cooperatividad energética característica de las conformaciones helicoidales se suele acompañar de momentos dipolares inducidos, los cuales son fundamentalmente debidos a la extensión espacial de interacciones tipo dipolo-dipolo ${ }^{[36]}$.

\section{Propiedades ópticas nos lineales en polímeros conjugados}

Las propiedades ópticas no lineales de las moléculas y polímeros conjugados es de suma importancia para el uso de estos materiales en dispositivos fotónicos. Estas propiedades, entre las que destacan la polarizabilidad $(<\alpha>)$, la primera hiperpolarizabilidad $(<\beta>)$ y la segunda hiperpolarizabilidad $(\langle\gamma\rangle)$, no son más que la respuesta de las moléculas a radicación óptica electromagnética. Las propiedades ópticas no lineales son sensibles a muchos factores estructurales, como por ejemplo la extensión de la conjugación $^{[39]}$, los sustituyentes donadores y/o aceptores de electrones ${ }^{[40]}$, la presencia de defectos ${ }^{[41]} \mathrm{o}$ dopantes ${ }^{[42]} \mathrm{y}$ la conformación molecular ${ }^{[43]}$. En consecuencia, el conocimiento de las relaciones entre la estructura y las propiedades ópticas no lineales son esenciales para el diseño de nuevos materiales.

Estudios teóricos basados en cálculos de mecánica cuántica han puesto de manifiesto que el dopado de algunos polímeros conjugados conlleva el realce de propiedades tales como la $\langle\alpha\rangle$ y $\langle\beta\rangle^{[44-46]}$. Al dopar una cadena finita de polímero se origina una molécula ionizada con la correspondiente distorsión estructural en las distancias de enlace. Esta distorsión se ha estudiado mediante cálculos ab initio en oligómeros constituidos por unos pocos anillos de tiofeno ${ }^{[47]}$. La estructura electrónica de estos compuestos en estado neutro es de tipo benzoideo y se caracteriza por una secuencia alternante y regular de enlaces $\mathrm{C}-\mathrm{C}$ y $\mathrm{C}=\mathrm{C}$ a lo largo de la cadena de polímero. $\mathrm{Al}$ interaccionar con un dopante, se transfieren electrones dando lugar a especies cargadas de naturaleza polarónica (catión radical) o bipolarónica (dicatión), cuya estructura electrónica es de tipo quinoideo. Además, cálculos semiempíricos en politiofeno indicaron que estos defectos de carga se extienden espacialmente a lo largo de la cadena polimérica, lo cual se refleja en que las distancias de enlace $\mathrm{C}-\mathrm{C}$ y $\mathrm{C}=\mathrm{C}$ características de la estructura quinoidea son diferentes en anillos consecutivos $^{[48,49]}$. La Figura 4 muestra la variación secuencial de las distancias de enlace entre átomos de carbono, calculadas mediante el métodos semiempírico AM1, a lo largo de una cadena de formada por 18 anillos de tiofeno en estados neutro y bipolarónico. Puede observarse que esta metodología cuántica reproduce correctamente las estructuras electrónicas de estas especies (benzoidea y quinoidea, respectivamente), cuyas características más signifcativas son: i) las posiciones de los enlaces $\mathrm{C}-\mathrm{C}$ y $\mathrm{C}=\mathrm{C}$ están invertidas en la estructura benzoidea y quinoidea; ii) las distancias de enlace presentan una gran regularidad espacial en la estructura benzoidea; iii) el defecto de carga se extiende a un número considerable de anillos, los cuales están ubicados en el centro de la cadena de polímero; y iv) en el bipolarón los extremos de la cadena presentan una estructura de tipo benzoideo, lo cual indica que la extensión espacial del defecto es limitada.

Por otro lado, cálculos cuánticos a nivel semiempírico han puesto de manifiesto que tanto $\langle\alpha\rangle$ como $\langle\gamma\rangle$ están muy influenciadas por los electrones conjugados $\pi$. En consecuencia los valores máximos de dichas propiedades se obtienen para la conformación planar anti en que la conjugación es máxima ${ }^{[43]}$. Por ejemplo, la $<\alpha>$ calculada para la conformación anti del bitiofeno es aproximadamente 102.5 u.a., mien-
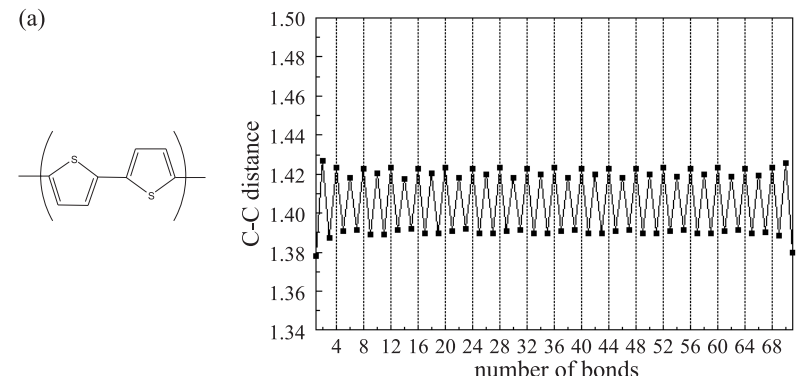

(b)

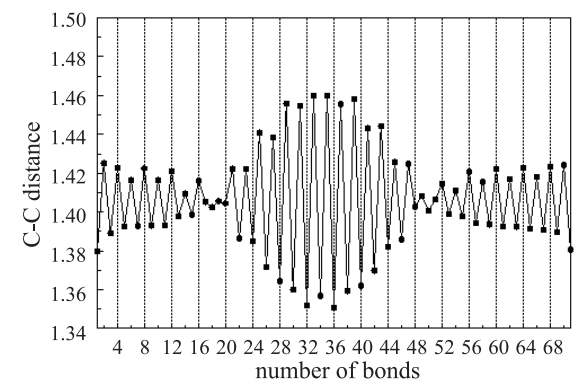

Figura 4. Variación secuencial de las distancia de enlace entre átomos de carbono a lo largo de una molécula formada por 18 anillos de tiofeno en estado neutro (a) y bipolarónico (b). La estructura electrónica característica de cada uno de dichos estados (benzoidea y quinoidea, respectivamente) se muestra en el esquema. 
tras que este valor disminuye casi dos unidades cuando los dos anillos están dispuestos perpendicularmente (deslocalización electrónica mínima).

Obviamente, las propiedades ópticas no lineales también dependen del tamaño molecular. Cálculos teóricos han ilustrado esta dependencia evaluando las diferencias de $\langle\alpha\rangle$ entre oligómeros consecutivos, lo cual se suele expresar mediante:

$$
\Delta<\alpha>=\langle\alpha\rangle_{\mathrm{N}}-\langle\alpha\rangle_{\mathrm{N}-1}
$$

donde $N$ corresponde al número de unidades químicas repetitivas presentes en el oligómero. Cálculos $a b$ initio HF indicaron que $\Delta<\alpha\rangle$ es $58.7,69.5$ y 77.0 u.a. cuando el oligómero contiene 2, 3 y 4 anillos de tiofeno, respectivamente ${ }^{[50]}$. Por otro lado, los resultados obtenidos para oligómeros mayores indicaron que este crecimiento se satura para 7 anillos de tiofeno y $\Delta<\alpha>$ converge asintóticamente a $85.1 \pm 1.9$ u.a. Es evidente que el estudio sistemático de propiedades ópticas no lineales mediante metodologías teóricas resulta de gran interés el diseño de materiales óptimos que, sin lugar a dudas, es el objetivo fundamental de la Ingeniería Molecular.

\section{Determinación de la estructura y plegamiento lamelar en poliamidas convencionales (nylons)}

Las técnicas de simulación basadas en la mecánica clásica han permitido abordar de forma eficiente el estudio no sólo de la estructura de los nylons ${ }^{[51-55]}$, sino también el mecanismo de plegamiento de las cadenas en las lamelas. Un adecuado ejemplo de ello lo constituye el nylon 4,6:<smiles>CNCCCCNC(=O)CCCCC(C)=O</smiles>

Nylon 4,6

Muy probablemente el nylon 4,6 sea el miembro más relevante del grupo de los nylons $2 n, 2 n+2$, ya que se trata de una de las poliamidas comerciales más ampliamente utilizadas. Este polímero se caracteriza por tener una cristalinidad muy elevada, un punto de fusión considerablemente alto, y unas propiedades físicas excelentes, superiores por ejemplo a las del nylon 6,6. Sin embargo, aunque se propuso una conformación extendida para las cadenas, la estructura cristalina de este polímero no se pudo determinar a partir de la información experimental disponible.

La Figura 5 muestra los modelos posibles para la estructura cristalina del nylon 4,6. Los puentes de hidrógeno pueden formarse en la dirección normal $(n)$ a la lámina o en dirección oblicua $(o)$ (Figura 5a). Las diferentes láminas pueden disponerse de manera que las cadenas adyacentes estén dispuestas de forma paralela (modelo I) o antiparalela (modelo II) (Figura 5b). Finalmente, las láminas pueden presentar un desplazamiento alternado (modelo $\alpha$ ) o progresivo (modelo $\beta$ ) en la dirección del eje c (Figura $5 \mathrm{c}$ ). La energía para todos los modelos posibles del nylon 4,6 se calculó considerando diferentes valores para el desplazamiento entre láminas en la dirección (a)

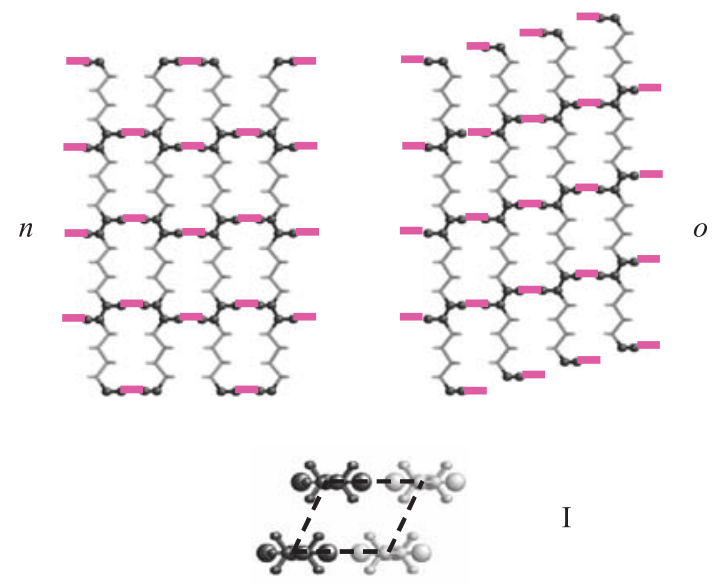

(b)

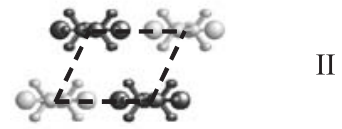

II

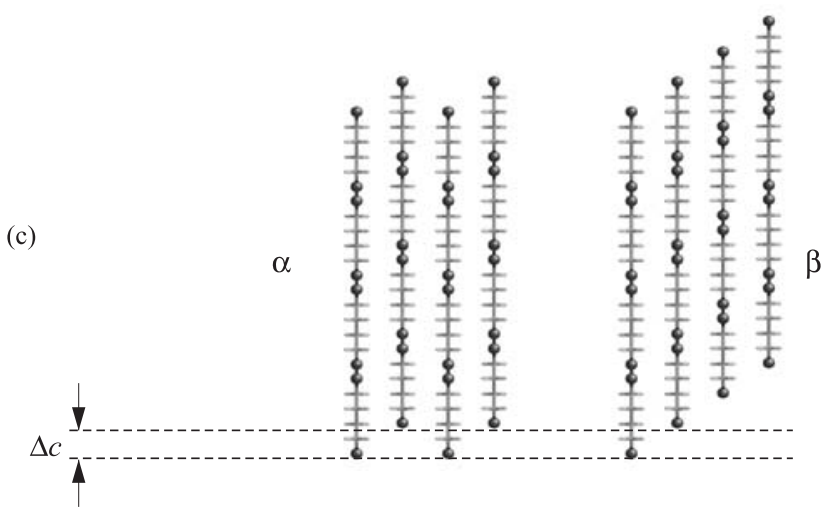

Figura 5. Modelos posibles para la estructura cristalina del nylon 4,6: (a) esquemas de puentes de hidrógeno normal (n) y oblicuo (o); (b) modelos paralelo (I) y antiparalelo (II); (c) modelos alternado $(\alpha)$ y progresivo ( $\beta$ ).

del eje $c$ (Figura 6) ${ }^{[54,55]}$. Para ello se empleo un potencial clásico análogo al descrito en las ecuaciones 2, 3 y 4.

Los modelos constituidos por un esquema de puentes de hidrógeno oblicuo son energéticamente poco estables. Por otro lado, los modelos de tipo II están considerablemente desfavorecidos con respecto a los de tipo I, independientemente de que el desplazamiento de las láminas sea alternado o progresivo. En los empaquetamientos constituidos por un esquema de puentes de hidrógeno normal y un apilamiento de láminas de tipo I se observa que, las diferencias de energía entre los modelos $\alpha$ y $\beta$ por un lado, y entre los desplazamientos de $2.33 c / 12$ y $3.66 c / 12$ unidades por otro, son pequeñas. Este hecho sugiere para el nylon 4,6 la existencia de una estructura estadística constituida por una mezcla de modelos $\alpha$ y $\beta$ con dos valores posibles de desplazamientos entre láminas para cada uno de ellos ${ }^{[54]}$. Dicha estructura, la cual se representa de forma esquemática en la Figura 7, proporciona una descripción satisfactoria de diferentes observaciones experimentales, tales como los diagramas de difracción de rayos $\mathrm{X}$.

Por otro lado, el mecanismo del plegamiento lamelar para el nylon 4,6 se investigó mediante simulaciones de dinámica 


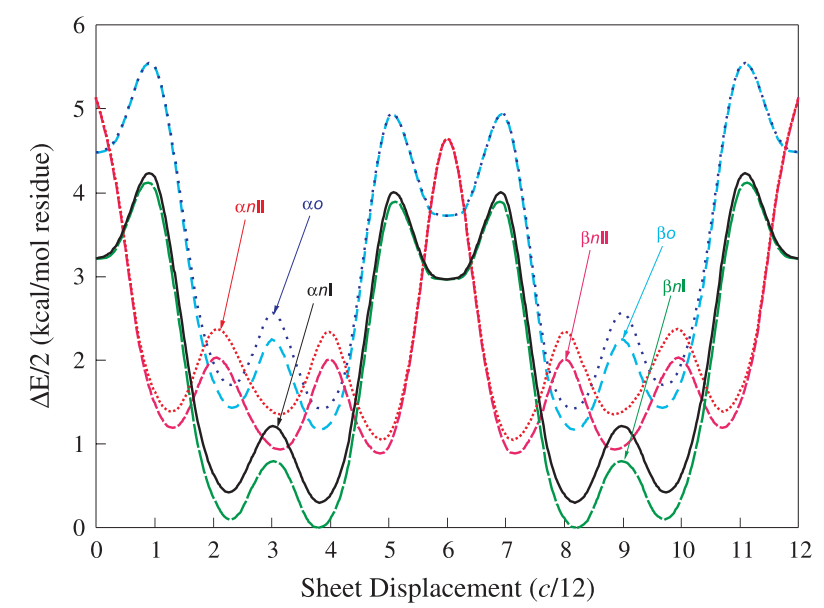

Figura 6. Perfiles energéticos obtenidos para los modelos posibles del nylon 4,6. Se representa la energía (en $\mathrm{kcal} / \mathrm{mol}$ residuo) en función del desplazamiento entre láminas en la dirección del eje $c$ (en unidades de c/12).
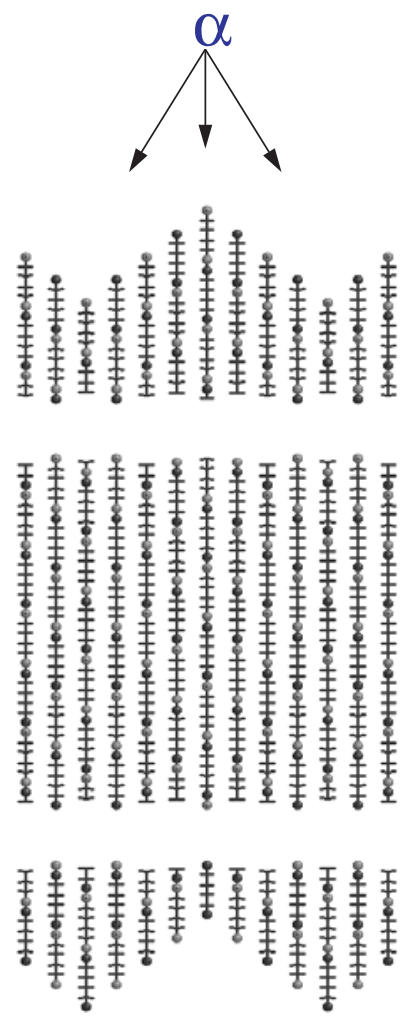

Figura 7. Representación esquemática de la lamela propuesta para el nylon 4,6, la cual está constituida por una mezcla de estructuras $\alpha$ y $\beta$.

molecular ${ }^{[56]}$. Para ello se considero que el plegamiento lamelar tiene lugar entre cadenas adyacentes de una misma lámina. Existe un total de cuatro modelos de plegamiento posibles para este polímero (Figura 8): dos que incluyen un grupo amida dentro del pliegue $\left(\mathrm{C}_{14} \mathrm{y} \mathrm{C}_{16}\right)$ y dos que incluyen los segmentos polimetilénicos del diácido y la diamina $\left(\mathrm{C}_{9 \mathrm{C}}\right.$ y $\left.\mathrm{C}_{9 \mathrm{~N}}\right)$. Se construyeron cristales de nylon 4,6 considerando cada uno de dichos modelos de plegamiento, cuya estabilidad energética fue analizada a partir de las trayectorias que describen a lo largo del tiempo ${ }^{[56]}$. El modelo $\mathrm{C}_{14}$ resultó ser el energéticamente más favorecido indicando que el ple- (a)
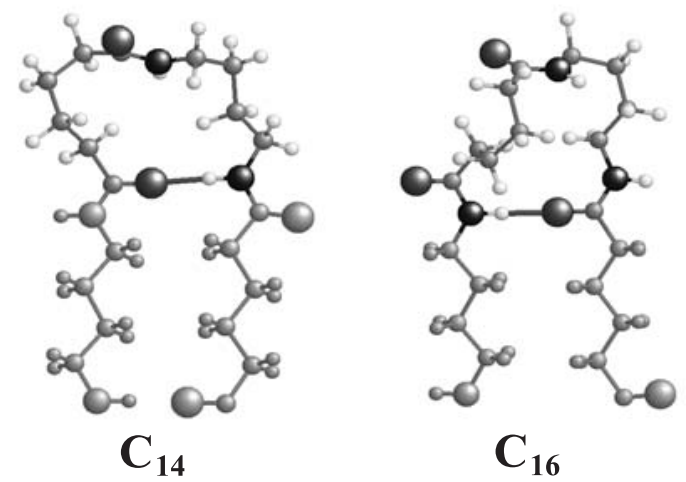

(b)

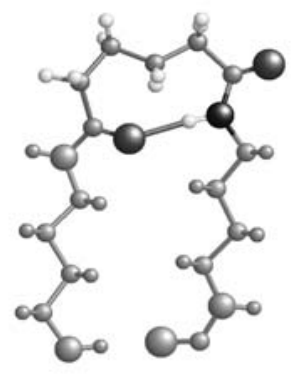

$\mathrm{C}_{9 \mathrm{C}}$

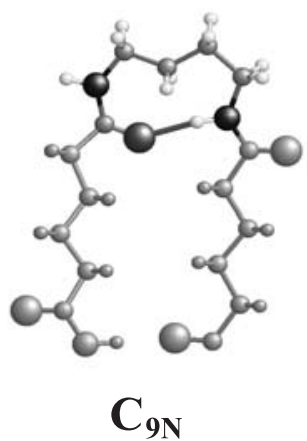

Figura 8. Modelos posibles para el plegamiento de una cadena de nylon 4,6: (a) modelos que incluyen un grupo amida dentro del pliegue, y (b) modelos que se pliegan por el segmento polimetilénico. La nomenclatura empleada para cada modelo hace referencia al número de átomos en el ciclo generado por el puente de hidrógeno involucrado en el plegamiento.

gamiento lamelar en el nylon 4,6 incluye un grupo amida. Recientemente se ha reportado en la literatura un estudio de características similares, en el cuál también se emplean técnicas de dinámica molecular, para estudiar el plegamiento lamelar del nylon $6^{[57]}$.

\section{Análisis de las interacciones específicas en complejos polielectrolito-tensioactivo derivados de polipéptidos}

Existe un gran número de materiales cuya obtención se basa únicamente en la capacidad que presentan sus componentes moleculares para dar asociaciones estables. En estos sistemas, los cuales se denominan de forma general complejos autoasociados, los distintos componentes se unen mediante interacciones de tipo no covalente, como puentes de hidrógeno, interacciones hidrofóbicas o interacciones electrostáticas. De todos los materiales formados por autoasociación, los más extensamente estudiados son aquellos que están constituidos por cadenas poliméricas cargadas (polielectrolitos) e iones moleculares anfifílicos (tensioactivos). En particular, los polielectrólitos poliméricos derivados de polipéptidos asociados con tensioactivos formados por pequeñas cabezas polares y una cola hidrofóbica han despertado gran interés (Figura 9a ${ }^{[58]}$.

Los complejos polipeptido tensioactivo más ampliamente estudiados han sido aquellos constituidos por el ácido poli $(\alpha$-glutámico) (PAGA) y tensioactivos del tipo alquiltrimetilamonio (ATMA) ${ }^{[58]}$, a los que se denominará ATMA.PAGA (Figura 9a). Estos compuestos se caracterizan por la tendencia a adoptar estructuras ordenadas tanto en 
estado sólido como en disolventes orgánicos. Así, en los complejos ATMA.PAGA las cadenas de polipéptido adoptan una conformación de hélice $\alpha$ similar a la esquematizada en la Figura $9 b^{[59,60]}$. Estudios computacionales basados en técnicas tanto cuánticas como clásicas han permitido profundizar en determinados aspectos microscópicos de este tipo de complejos autoasociados ${ }^{[61-64]}$.

Cálculos mecanocuánticos usando el método MP2 indicaron que la energía libre de unión implicada en la interacción electrostática característica de los complejos ATMA·PAGA es de $-84 \mathrm{kcal} / \mathrm{mol}^{[61,62]}$. Además, esta interacción resulta

(a)

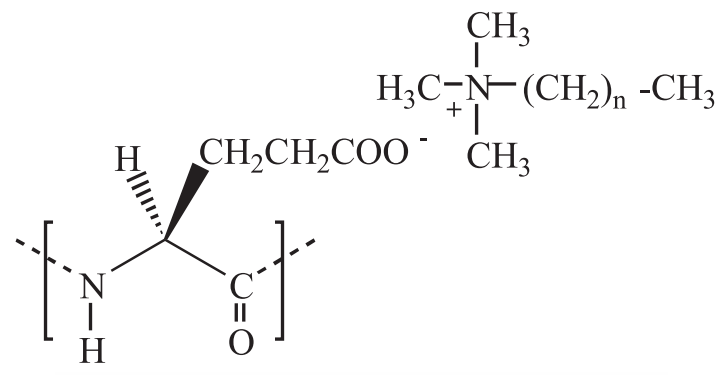

(b)

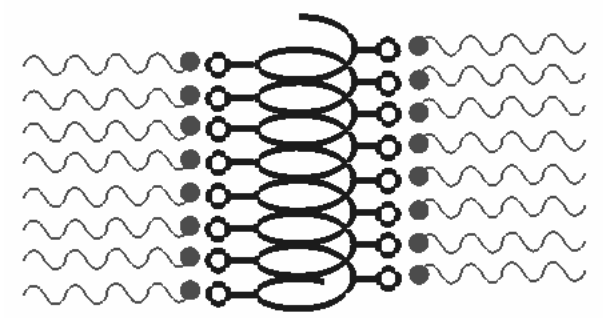

(c)

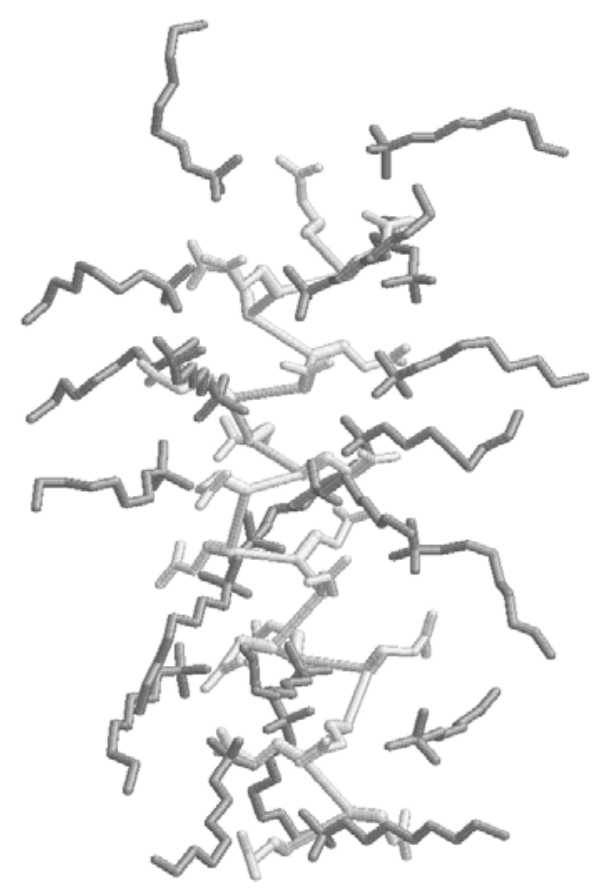

Figura 9. Representación esquemática de la estructura química (a) y molecular (b) de los complejos ATMA·PAGA. (c) Proyección axial de una estructura representativa obtenida mediante simulaciones de dinámica molecular del complejo ATMA.PAGA en disolución de cloroformo. Con el objetivo de clarificar la imagen las cadenas laterales se describen mediante esferas y los tensioactivos no se representan. alrededor de $2 \mathrm{kcal} / \mathrm{mol}$ más estable cuando la cadena alquílica del catión molecular tiene pocos átomos de carbono. Por otro lado, las técnicas cuánticas revelaron que la formación de estos complejos en disolución acuosa está desfavorecida debido al gran coste energético que implica la desolvatación de iones moleculares no acomplejados ${ }^{[61]}$. Este coste energético es menor en disolventes orgánicos como el cloroformo, siendo parcialmente compensado por la fuerte interacción electrostática entre los iones moleculares. Estudios mecanocuánticos en sistemas modelo correspondientes a otras familias de complejos autoasociados han proporcionado resultados similares ${ }^{[65,66]}$

Por otro lado, simulaciones de dinámica molecular de complejos ATMA.PAGA en disolución de cloroformo permitieron racionalizar la gran estabilidad conformacional de la hélice $\alpha$ que presenta la cadena polipeptídica ${ }^{[63,64]}$. En particular, las trayectorias obtenidas permitieron observar que las cadenas de tensioactivo impiden a las moléculas de disolvente interaccionar con el polipéptido. Además, los grupos polares del polipéptido no pueden interaccionar con los grupos carboxilato laterales, ya que estos últimos están continuamente interaccionando con el tensioactivo (Figura 9c). Este comportamiento contrasta con el observado en las si-

(a)

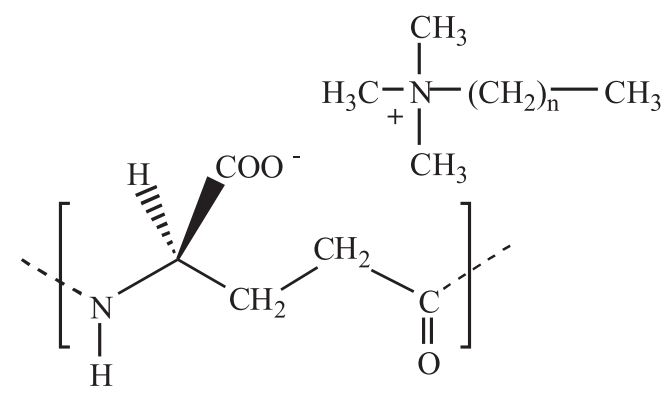

(b)

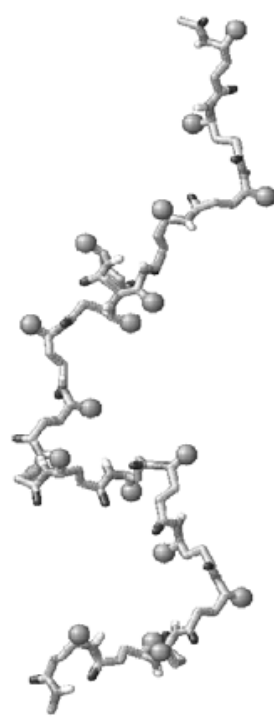

Figura 10. (a) Representación esquemática de la estructura química de los complejos autoasociados derivados del ácido poli( $\gamma$-glutámico). (b) Conformación de la cadena de polielectrolito que resulta de las simulaciones de dinámica molecular de los complejos autoasociados derivados del ácido poli( $\gamma$-glutámico). Con el objetivo de clarificar la imagen las cadenas laterales se describen mediante esferas y los tensioactivos no se representan. 
mulaciones de dinámica molecular de otros complejos en los que el polielectrolito no está constituido por $\alpha$-amino acidos, como por ejemplo polielectrolitos derivados del ácido poli $(\alpha$-glutámico $)$-el isómero constitucional del ácido poli( $\alpha$-glutámico) (Figura 10a)-. En estos sistemas el polielectrolito pierde su estructura secundaria (Figura 10b) como consecuencia de la interacción entre los tensioactivos y los grupos polares de la cadena principal, la cual es debida a la mayor accesibilidad de esta última.

\section{Análisis de parámetros espectroscópicos obtenidos mediante Resonancia Magnética Nuclear (RMN)}

Los parámetros obtenidos a partir de espectroscopía de RMN, las constantes de acoplamiento (J) y los desplazamientos químicos $(\sigma)$, están directamente relacionados con la conformación molecular local y el entorno químico más próximo. De hecho esta técnica espectroscópica es insensible a efectos de largo alcance. Esta característica resulta de gran importancia a la hora de intentar reproducir y/o analizar los parámetros espectroscópicos mediante metodologías teóricas, ya que permite que reducir la simulación a un pequeño sistema modelo. En consecuencia, en muchos casos no es preciso recurrir a técnicas clásicas para estudiar los parámetros de RMN, ya que el sistema considerado es perfectamente abordable mediante técnicas cuánticas.

La constante de acoplamiento ${ }^{3} \mathrm{~J}_{\mathrm{NH}-\mathrm{CH}}$ para el estado de ovillo estadístico de los poli( $\alpha$-alquil- $\beta$-L-aspartato $)$ se pudo simular teóricamente a partir de las preferencias conformacionales del dipéptido modelo $\alpha$-metilo- $\alpha$-L-aspartamilo ${ }^{[67]}$. Así, se asumió que un polímero en estado de ovillo estadístico no presenta interacciones locales específicas entre diferentes residuos, y se puede modelar considerando únicamente las preferencias conformacionales de los residuos que lo constituyen. En consecuencia basta con realizar un análisis exhaustivo de los mínimos de energía del compuesto modelo correspondiente a partir de métodos de cálculo basados en la mecánica cuántica ${ }^{[68]}$, lo cual es mucho más rápido y sencillo que investigar un estado desordenado para el polímero completo mediante dinámica molecular. Los ángulos diedros de los mínimos de energía del compuesto modelo pueden relacionarse a partir de sencillas formulas matemáticas con las constantes de acoplamiento. En el caso de los poli $(\alpha$-alquil- $\beta$-L-aspartato) la constante de acoplamiento ${ }^{3} \mathrm{~J}_{\mathrm{NH}-\mathrm{CH}}$ obtenida a partir de este procedimiento teórico, 7.9 $\mathrm{Hz}$, presentó una buena concordancia con la determina experimentalmente, $8.5 \mathrm{~Hz}^{[67]}$.

Por otro lado, la simulación de desplazamientos quími$\cos , \sigma$, requiere de métodos cuánticos ${ }^{[69]}$ que permitan determinar el valor esperado de la energía con respecto a un campo magnético externo B y el momento nuclear magnético $\mu$ del núcleo $\mathrm{M}$ en cuestión:

$$
\sigma_{\alpha \beta}^{M}=\frac{\partial^{2} E}{\partial \mu_{\alpha}^{M} \partial B_{\beta}} \alpha, \beta=x, y, z
$$

Tabla 2. Comparación entre los desplazamientos químicos (en ppm) de ${ }^{13} \mathrm{C}, \delta\left({ }^{13} \mathrm{C}\right)$, obtenidos experimentalmente y mediante cálculos DFT para las formas $\alpha$ y $\gamma$ del nylon 6 .

\begin{tabular}{cccccccc}
\hline Forma & $\#$ & C1 & C2 & C3 & C4 & C5 & C6 \\
\hline \multirow{2}{*}{$\alpha$} & Experim. & 173.4 & 36.7 & 26.5 & 30.4 & 30.4 & 43.6 \\
& DFT & 171.8 & 38.4 & 30.1 & 32.4 & 33.1 & 44.3 \\
& Experim. & 173.0 & 37.8 & 30.1 & 30.1 & 34.1 & 39.9 \\
$\gamma$ & DFT & 173.4 & 38.6 & 31.8 & 33.5 & 36.9 & 40.3 \\
\hline
\end{tabular}

Sin embargo, el número de estudios dedicados predicción de $\sigma$ es aún relativamente escaso, ya que muchos de los procedimientos químico-cuánticos requeridos para ello fueron desarrollados hace relativamente poco tiempo ${ }^{[69]}$. Este número es aún más reducido en el caso de sistemas poliméricos, lo cuál es debido a una complicación adicional: el uso de técnicas cuánticas obliga a describir estos sistemas de naturaleza macromolecular mediante modelos reducidos, pero estos últimos deben ser elegidos de forma que representen correctamente la microestructura de los primeros ${ }^{[70-72]}$.

La Tabla 2 compara los desplazamientos químicos de ${ }^{13} \mathrm{C}$, $\delta\left({ }^{13} \mathrm{C}\right)$, determinados experimentalmente y a partir de cálculos DFT para las formas $\alpha$ y $\gamma$ del nylon 6 (Figura 11). Los parámetros teóricos fueron obtenidos considerando un pequeña sistema modelo constituido por tres cadenas de nylon 6 interaccionando por puentes de hidrógeno ${ }^{[70]}$. La bondad de los resultados pone de manifiesto la sensibilidad de este tipo de cálculos a la fenómenos tales como la conformación molecular y las interacciones intermoleculares. De hecho, la simulación de los desplazamientos químicos observados experimentalmente pueda considerarse como una herramienta prometedora, y complementaria a la cristalografía, para estudiar la estructura de los polímeros cristalinos.

Hay que mencionar que las técnicas teóricas permiten reproducir la información experimental obtenida a partir de otros tipos de espectroscopías. Por ejemplo, se puede determinar los modos normales de vibración (frecuencia e intensidad) característicos de los espectroscopía i.r. ${ }^{[73-76]}$, las constantes de acoplamiento de E.P.R ${ }^{[77,78]}$, etc...

\section{Estudio de la solubilidad de gases en matrices poliméricas}

La permeabilidad de gases en matrices poliméricas depende de dos factores: la solubilidad del gas en el polímero, es decir, la cantidad de moléculas de gas que puede ser absorbida y la difusión de dichas moléculas en el interior de la matriz. Uno de los conceptos más importantes en el estudio de procesos de absorción de gases en sistemas poliméricos es el de espacio accesible ${ }^{[79,80]}$. Para que un soluto pueda disolverse en el interior de un polímero es necesario que exista espacio para que este pueda penetrar en su interior. Así, la fracción de volumen que no está ocupada por los átomos de las cadenas de polímero se define como fracción de espacio accesible.

Para poder simular procesos de solubilidad es necesaria una descripción atomística de la matriz polimérica estudiada ${ }^{[81,82]}$. 
(a)

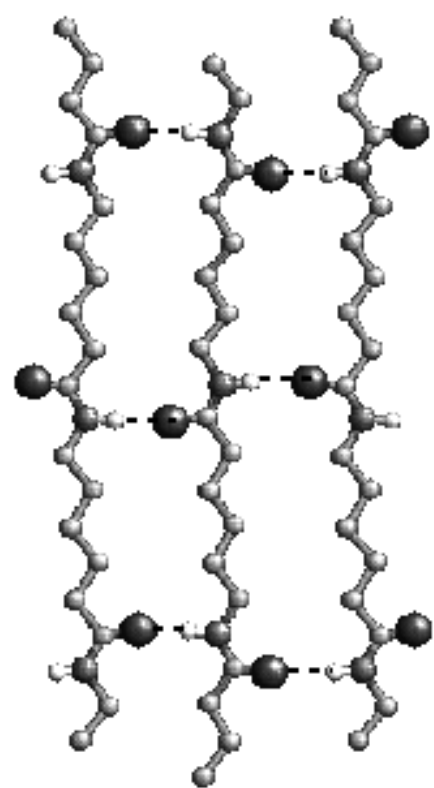

(b)

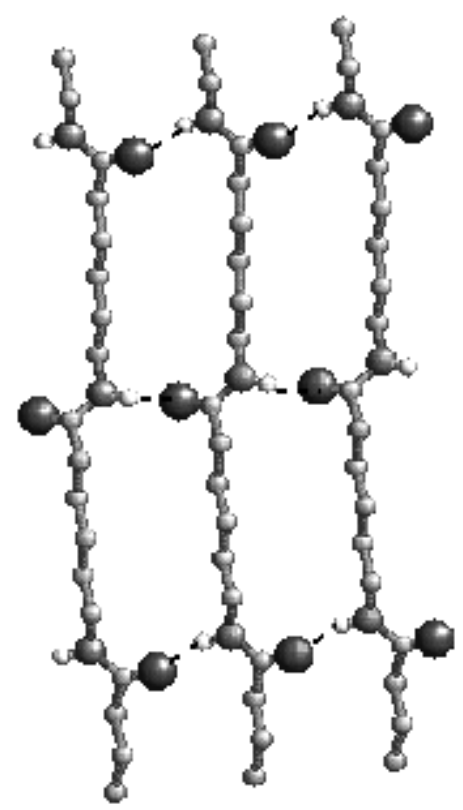

Figura 11. Representación esquemática de las cadenas enlazadas por puente de hidrógeno en las formas $\alpha$ (a) y $\gamma$ (b) del nylon 6 .

Es decir, se deben obtener mediante simulaciones de dinámica molecular o Monte Carlo un número de microestructuras representativas suficientemente amplio para que en conjunto proporcionen una descripción adecuada de la matriz. La determinación del espacio accesible en cada una de dichas microestructuras permitirá calcular de forma eficiente el exceso potencial químico $\left(\mu^{\mathrm{ex}}\right)$ que se produce al insertar una molécula de gas en la matriz. El $\mu^{\mathrm{ex}}$ está directamente relacionado con la solubilidad del gas a dilución infinita, y depende de la energía de interacción entre dicha molécula y el polímero $(\phi)$ :

$$
\begin{aligned}
& \beta \mu^{\mathrm{ex}}(\rho, \mathrm{T})=-\ln \langle\exp (-\beta \phi)\rangle \\
& \mathrm{S}=\left\{\exp \left(\mu^{\mathrm{ex}} / \mathrm{RT}\right)\right\} / \mathrm{RT}
\end{aligned}
$$

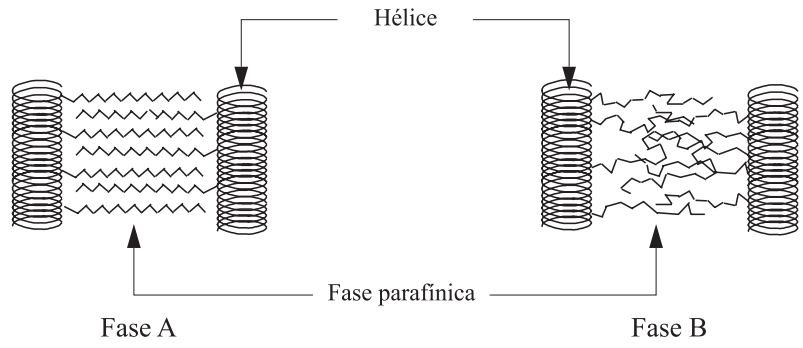

Figura 12. Representación esquemática de las fases A y B de los polímeros tipo peine capaces de adoptar conformaciones helicoidales.

donde $\beta=1 / \mathrm{k}_{\mathrm{b}} \mathrm{T}, \mathrm{k}_{\mathrm{b}}$ y $\mathrm{T}$ son la constante de Boltzmann y la temperatura y $\rho$ es la densidad específica del sistema.

Los estudios computacionales relacionados con la solubilidad y la permeabilidad de gases polímeros habitualmente se ciñen a la fase amorfa de la matriz ${ }^{[82]}$. Ello se debe a que la movilidad de los penetrantes es mucho mayor en la fase amorfa que en la cristalina, ya que el empaquetamiento de las cadenas es mucho menos eficiente en esta última. En consecuencia, se ha aceptado tradicionalmente que el transporte de gases en polímeros semicristalinos ocurre fundamentalmente en la fase amorfa. Una excepción a esta regla general es el poli(4-metil-1-penteno), el cual presenta solubilidades y permeabilidades muy parecidas en ambas fases. Ello se debe a que la densidad en la fase cristalina es muy parecida a la de la fase amorfa. En consecuencia, la solubilidad de gases en la fase cristalina del PMP ha sido objeto de varios estudios de simulación molecular, empleándose para ello métodos de dinámica molecular ${ }^{[83]}$ y Monte Carlo ${ }^{[84]}$.

Otros materiales que presentan un comportamiento excepcional son los polímeros tipo peine constituidos por una cadena principal capaz de adoptar una conformación rígida, (habitualmente una hélice) y cadenas laterales largas de naturaleza alquílica. Estos sistemas adoptan estructuras bifásicas en las que las hélices se empaquetan en láminas, mientras que las cadenas laterales permanecen ocupando el espacio inter-laminar (Figura 12). En función de la temperatura, las cadenas alquílicas pueden estar desordenadas de manera que no exista estructuración alguna en la fase parafínica (fase B) o cristalizadas en una red de tipo hexagonal (fase A). Estudios recientes han puesto de manifiesto que la solubilidad y permeabilidad de gases en este tipo de polímeros cambia drásticamente con la temperatura, es decir con la estructura de la fase parafínica ${ }^{[85]}$. Así, los coeficientes medidos cuando las cadenas alquílicas están desordenadas son mucho más altos que los obtenidos cuando la fase parafínica esta cristalizada. Ello indica que, aún a pesar de su orden laminar, los polímeros tipo peine son potencialmente interesantes para el desarrollo de membranas.

Recientemente, se estudió mediante simulaciones de Monte Carlo la solubilidad de diferentes gases $\left(\mathrm{He}, \mathrm{Ar}\right.$ y $\left.\mathrm{CH}_{4}\right)$ en diferentes matrices constituidas por PAALAs con cadenas alquílicas laterales largas, $\mathrm{R}=-\left(\mathrm{CH}_{2}\right)_{\mathrm{n}-1}-\mathrm{CH}_{3}$ con $\mathrm{n}=12,14 \mathrm{y}$ $16^{[86]}$. Dichos polímeros se estructuraron en láminas formadas por hélices 13/4 que estaban separadas por una fase parafínica desordenada (Figura 13). La Tabla 3 muestra la cantidad de 


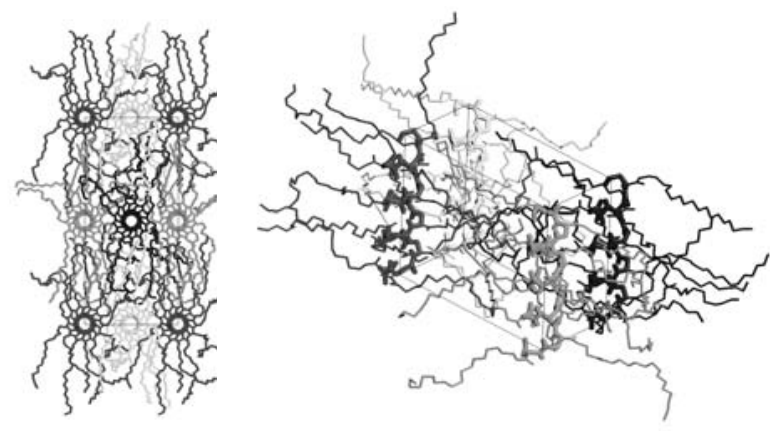

Figura 13. Proyección basal y en perspectiva de la estructura bifásica de los PAALAs con cadenas laterales largas.

Tabla 3. Espacio accesible (en \%) y exceso de potencial químico (entre paréntesis y en $\mathrm{kcal} / \mathrm{mol}$ ) para diferentes gases en matrices poliméricas constituidas por PAALAs con diferentes cadenas laterales.

\begin{tabular}{lccc}
\hline \multicolumn{1}{c}{$\mathbf{R}$} & $\mathbf{H e}$ & $\mathbf{A r}$ & $\mathbf{C H} 4$ \\
\hline$-\left(\mathrm{CH}_{2}\right)_{11}-\mathrm{CH}_{3}$ & $4.1(-1.78)$ & $2.0(-1.20)$ & $1.4(-0.91)$ \\
$-\left(\mathrm{CH}_{2}\right)_{13}-\mathrm{CH}_{3}$ & $1.4(-1.89)$ & $0.4(-1.04)$ & $0.2(-0.61)$ \\
$-\left(\mathrm{CH}_{2}\right)_{15}-\mathrm{CH}_{3}$ & $0.8(-1.56)$ & $0.2(-0.48)$ & $0.1(0.06)$ \\
$-\left(\mathrm{CH}_{2}\right)_{3}-\mathrm{CH}_{3}$ & $2.4(-1.62)$ & $0.7(-0.91)$ & $0.0(-)$ \\
$-\mathrm{CH}_{3}$ & $0.6(-0.18)$ & $0.1(-)$ & $0.0(-)$ \\
\hline
\end{tabular}

espacio accesible y el $\mu^{\text {ex }}$ (ecuación 11) obtenidos para cada uno de los sistemas estudiados (ecuación 11). En la misma Tabla se listan los valores calculados para los PAALAs con cadenas laterales cortas, $n=1$ y $3^{[87]}$, los cuales adoptan estructuras ordenadas tridimensionalmente (Figura 3). Los resultados evidencian que el tipo de empaquetamiento, así como el grado de orden, influye de forma considerable en la capacidad de los PAALAs para absorber gases. Así, las moléculas de gas interaccionan de forma mucho más favorable con los polímeros con estructuras bifásicas que con los que presentan orden tridimensional. Estudios de simulación basados en técnicas avanzadas de Monte Carlo han permitido detectar un comportamiento análogo para los poli(alquil-acrilatos) tipo peine ${ }^{[88,89]}$. Estos resultados ponen de manifiesto la estrecha relación que existe entre las estructura de los materiales y sus propiedades.

\section{Predicción de propiedades mecánicas}

La predicción de propiedades mecánicas mediante el uso de metodologías teóricas ha sido, sin duda alguna, uno de los objetivos más ambiciosos de los químicos teóricos dedicados a la Ciencia de los Polímeros. El modelado del módulo cristalino, o módulo de Young, puede realizarse tanto mediante técnicas cuánticas como clásicas ${ }^{[90]}$. La aplicación de las primeras está restringida a compuestos modelo de pequeño tamaño mientras que las últimas permiten una descripción más completa del sistema, si bien en todos los casos el módulo se predice a partir de la variación de energía que induce la aplicación de una fuerza externa estática.

Las propiedades mecánicas de del poli(etilentereftalato), abreviado PET, han sido estudiadas mediante un gran número
Tabla 4. Módulo de Young (en Gpa) experimental y calculado para las dos formas cristalinas del PET.

\begin{tabular}{cccc}
\hline Metodología & Referencia & T1 & T2 \\
\hline Experimental & 94 & 74,110 & 86 \\
Simulación molecular & 73 & 169 & 141 \\
Simulación molecular & 91 & 192 & 217 \\
Simulación molecular & 92 & 96 & - \\
Simulación molecular & 93 & 178 & \\
\hline
\end{tabular}

de técnicas de simulación molecular ${ }^{[73,91-93]}$. Ello se debe a la disponibilidad de valores experimentales ${ }^{[94,95]}$ para sus dos formas cristalinas, las cuales consisten en un empaquetamiento triclínico de cadenas en conformación extendida $\left(\mathrm{T}_{1}\right)$ y una fase mesomórfica en que las cadenas están ligeramente plegadas con respecto a la conformación anterior $\left(\mathrm{T}_{2}\right)^{[94,95]}$.

En la Tabla 4 se muestran los módulos de Young calculados para las formas $\mathrm{T}_{1}$ y $\mathrm{T}_{2}$ del PET mediante potenciales clásicos. Las discrepancia entre los módulos teóricos estimados por los diferentes autores son fundamentalmente debidas a: i) diferencias en los parámetros requeridos para calcular las diferentes contribuciones energéticas (ecuaciones 3 y 4 ); y/o ii) la aplicación de expresiones alternativas a los mostrados en la sección anterior para describir los términos energéticos enlazante y no-enlazante . En consecuencia puede deducirse que la predicción de propiedades mecánicas depende en gran medida del método teórico empleado. Por otro lado, la comparición entre los valores experimentales y calculados indica que los últimos están notablemente sobreestimados $(\sim 50 \%)$ con respecto a los primeros. Este es un resultado razonable, y de hecho esperado, ya que los cálculos teóricos se realizaron suponiendo un material 100\% cristalino sin ningún tipo de defecto en la orientación de los cristales. A pesar de estas limitaciones, es importante destacar que los cálculos reproducen los resultados experimentales desde un punto de vista cualitativo.

\section{Conclusión}

En este trabajo se han revisado los conceptos básicos relacionados con la utilización de métodos computacionales al estudio de la estructura y propiedades de polímeros. La aplicabilidad de las técnicas cuánticas y clásicas expuestas en él se ha ilustrado examinando la bondad y sensibilidad de dichas metodologías en la predicción de muchas y variadas propiedades.

En la actualidad las técnicas de simulación mostradas en este trabajo constituyen una herramienta de trabajo tremendamente poderosa para el estudio de sistemas químicos. Así, los métodos teóricos se emplean hoy en día de forma rutinaria para, por un lado explicar y racionalizar a escala atómica fenómenos y procesos que se han detectado experimentalmente, y por otro predecir las propiedades de sistemas de los que se carece de información experimental. 
En un futuro próximo la aplicabilidad de los métodos teóricos al estudio de los polímeros posiblemente será muy superior a la actual. En este sentido es importante destacar que en los últimos años están apareciendo nuevas metodologías teóricas, denominadas de coarse-grained, cuyo objetivo fundamental es estudiar fenómenos y procesos a escala mesoscópica ${ }^{[98]}$. Así, las combinación de los métodos atomísticos actuales junto con los de coarse-grained permitirá abarcará todo aquello que sucede entre los niveles atómico $(\sim 1 \AA)$ y mesocópico $(\sim 1 \mu \mathrm{m})$. Es de esperar que la aplicabilidad de estos métodos se consolide en un futuro inmediato.

\section{Agradecimientos}

Los autores están agradecidos a la DGICYT por la financiación del proyecto BQU2000-0990; al Centro de Supercomputación de Cataluña (CESCA) y al Centro Europeo de Paralelelismo de Barcelona (CEPBA) por las facilidades comutacionales ofrecidas; al Dr. S. León y al Dr. D. Zanuy por la asistencia durante la preparación de las figuras.

\section{Referencias}

1. Szabo, A.; Ostlund, N. S. Modern Quantum Chemistry: Introduction to Advanced Electronic Structure Theory; Dover Publications, Inc.: Mineola, New York, (1996).

2. Dewar, M. J. S.; Zoebisch, E. G.; Healy, E. F.; Stewart, J. J. P. - J. Am. Chem. Soc. 107, 3902 (1985).

3. Stewart, J. J. P. - J. Comput. Chem. 10, 209, (1989).

4. Parr, R. G.; Yang, W. - Density Functional Theory of Atoms and Molecules; Oxford Univ. Press: Oxford (1989).

5. Bowen, J. P.; Allinger, N. L. - En Reviews in Computational Chemistry, Volume 2; Lipkowitz, K. B., Boyd, D. B. (Eds.); VCH Publishers, Inc.: New York, pp 81-99 (1991).

6. Cornell, W. D.; Cieplak, P.; Bayly, C. I.; Gould, I. R.; Merz, K. M.; Ferguson, M. D.; Spellmeyer, D. C.; Fox, T.; Caldwell, J. W.; Kollman, P. - J. Am. Chem. Soc. 117, 5179 (1995).

7. Allen, F. H.; Tildesley, D. J. Computer Simulation of Liquids; Clarendon Oxford, (1989).

8. Leontidis, E.; de Pablo, J. J.; Laso, M.; Suter, U. W. - Adv. Polym. Sci. 116, 283 (1994).

9. León, S.; Alemán, C.; Escalé, F.; Laso, M. J. - Comput. Chem. 22, 162 (2001).

10. Theodorou, D. N.; Suter, U. W. - Macromolecules 18, 1467 (1985)

11. Clarke, J. H. R.; Brown, D. - Mol. Simul. 3, 27 (1989).

12. Müller-Plate, F.; Rogers, S. C.; van Gunsteren, W. F. - J. Chem. Phys. 98, 9895 (1993).
13. McKechnie, J. I.; Brown, D.; Clarke, J. H. R. Macromolecules, 25, 1562 (1992).

14. Gusev, A. A.; Zehnder, M. M.; Suter, U. W. Macromolecules, 27, 615 (1994).

15. van der Vegt, N. F. A.; Briels, W. J.; Wessling, M.; Strathmann, H. - J. Chem. Phys., 105, 8849 (1996).

16. Kotelyanskii, M.; Wagner, N. J.; Paulatis, M. E. Macromolecules, 29, 8497 (1996).

17. Müller, M.; Nievergelt, J.; Santos, S.; Suter, U. W. - J. Chem. Phys., 114, 9764 ( 2001).

18. Santos, S.; Suter, U. W.; Müller, M.; Nievergelt, J. - J. Chem. Phys., 114, 9772 (2001).

19. Curcó, D.; Alemán, C. - J. Chem. Phys., aceptado para publicación (2003).

20. Brisse, F. - J. Electron Microsc. Techn., 11, 272 (1989).

21. Sorensen, R. L.; Liau, W. B.; Kesner, L.; Boyd, R. H. Macromolecules, 21, 200 (1988).

22. Ferro, D. R.; Brückner, S.; Mielle, S. V.; Ragazzi, M. Macromolecules, 25, 5231 (1992).

23. Ferro, D. R.; Pravasoli, A. - Macromol. Theory Simul., 5, 231 (1996).

24. Wang, H.; Stubbs, G. - Acta Cryst., A49, 504 (1993).

25. León, S.; Navas, J. J.; Alemán, C. - Polymer, 40, 7351 (1999).

26. Ferro, D. R.; Porzio, W.; Destri, S.; Ragazzi, M. Macromol. Theory Simul., 6, 713 (1997).

27. Navas, J. J.; Alemán, C.; López-Carrasquero, F.; MuñozGuerra S. - Macromolecules, 28, 4487 (1995).

28. López-Carrasquero, F.; García-Alvarez, M.; Navas, J. J.; Alemán, C.; Muñoz-Guerra, S. - Macromolecules, 29, 8449 (1996).

29. García-Alvarez, M.; Martínez de Ilarduya, A.; León, S.; Alemán, C.; Muñoz-Guerra, S. - J. Phys. Chem. A, 101, 4215 (1997).

30. García-Alvarez, M.; León, S.; Alemán, C.; Campos, J. L.; Muñoz-Guerra, S. - Macromolecules, 31, 124 (1998).

31. Bella, J.; Alemán, C.; Fernández-Santín, J. M.; Alegre, C.; Subirana, J. A. - Macromolecules, 22, 5225 (1992).

32. Alemán, C.; Roca, R.; Luque, F. J.; Orozco, M. - Proteins, 28, 83 (1997).

33. Zanuy, D.; Alemán, C. - Biopolymers, 49, 497 (1999).

34. Alemán, C.; Navas, J. J.; Roca, R. - Biopolymers, 41, 721 (1997).

35. Seebach, D.; Ciceri, P. E.; Overhand, M.; Jaun, B.; Rigo, D.; Oberer, L.; Hommel, U.; Amstutz, R.; Widmer, H. - Helv. Chim. Acta, 79, 2043 (1996). 
36. Wu, Y.-D.; Zhao, Y.-L. - J. Am. Chem. Soc., 123, 5313 (2001).

37. Casanovas, J.; Alemán, C. - J. Comput. Aided Mol. Design, 8, 441 (1994).

38. Schafer, L.; Newton, S. Q.; Cao, M.; Peeters, A.; Van Alsenoy, C.; Wolinski, K.; Momany, F. A. - J. Am. Chem. Soc., 115, 272 (1993).

39. Mukamel, S.; Takahashi, A.; Wang, H. X.; Chen, G. Science, 266, 250 (1994).

40. Marder, S. R.; Torruellas, W. E.; Blanchard-Desce, M.; Ricci, V.; Stegemans, G. I.; Gilmour, S.; Bredas, J. L.; Li, J.; Bublitz, G. U.; Boxer, S. G. - Science, 276, 1233 (1997).

41. An, Z.; Wong, K. Y. - J. Chem. Phys., 114, 1010 (2001).

42. Champagne, B.; Spassova, M.; Jadin, J.; Kirtman, B. - J. Chem. Phys., 116, 3935 (2002).

43. Lukeš, V.; Breza, M.; Pálszegi, T.; Laurinc, V.; Vrábel, I. - Macromol. Theory Simul., 10, 592 (2001).

44. de Melo, C. P.; Fonseca, T. L. - Chem. Phys. Lett., 261, 28 (1996).

45. Luo, Y.; Norman, P.; Ågren, H. - J. Am. Chem. Soc., 120, 11188 (1998).

46. Luo, Y.; Norman, P.; Macak, P.; Ågren, H. - J. Chem. Phys., 111, 9853 (1999).

47. Alemán, C.; Juliá, L. - J. Phys. Chem., 100, 14661 (1996).

48. Ehrendorfer, Ch.; Karpfen, A. - J. Phys. Chem., 99, 5341 (1995).

49. Alemán, C. - Macromol. Theory Simul., 6, 237 (1997).

50. Champagne, B.; Jacquemin, D.; André, J. M. - J. Phys. Chem., 100, 2034 (1994).

51. Casas, M. T.; Armelin, E. A.; Alemán, C.; Puiggalí, J. Macromolecules, 35, 8781 (2002).

52. Wendoloski, J. J.; Gardner, K. H.; Hirschinger, J.; Miura, J.; English, A. D. - Science, 247, 431 (1990).

53. Dasgupta, S.; Hammond, W. B.; Goodard III, W. A. - J. Am. Chem. Soc., 118, 12291 (1996).

54. Bermúdez, M.; León, S.; Alemán, C.; Muñoz-Guerra, S. - J. Polym. Sci., 38, 41 (2000).

55. Bermúdez, M.; León, S.; Alemán, C.; Muñoz-Guerra, S. - Polymer, 41, 8961 (2000).

56. León, S.; Alemán, C.; Bermúdez, M.; Muñoz-Guerra, S. - Macromolecules, 33, 5754 (2000).

57. Li, Y.; Goddard III, W. A. - Macromolecules, 35, 8440 (2002).

58. MacKnight, W. J.; Ponomarenko, E. A.; Tirrell, D. A: Acc. Chem. Res., 31, 781 (1998).
59. Ponomarenko, E. A.; Waddon, A. J.; Bakeev, K. N.; Tirrell, D. A.; MacKnight, W. J. - Macromolecules, 29, 4343 (1996).

60. Ponomarenko, E. A.; Waddon, A. J.; Tirrell, D. A.; MacKnight, W. J. Langmuir 1996, 12, 2169.

61. Alemán, C.; Zanuy, D. - Chem. Phys. Lett., 319, 318 (2000).

62. Alemán, C.; Zanuy, D. - Chem. Phys. Lett., 343, 390 (2001).

63. Zanuy, D.; Alemán, C.; Muñoz-Guerra, S. - Biopolymers, 63, 151 (2002).

64. Zanuy, D.; Alemán, C. - Langmuir, 19, 3987 (2003).

65. Masella, M.; Cuniasse, P. - J. Chem. Phys., 119, 1866 (2003).

66. Alemán, C.; Zanuy, D.; Casanovas, J. - J. Phys. Chem., 107, 4151 (2003).

67. Martínez de Ilarduya, A.; Alemán, C.; García-Alvarez, M.; López-Carrasquero, F.; Muñoz-Guerra, S. Macromolecules, 32, 3257 (1999).

68. Navas, J. J.; Alemán, C.; Muñoz-Guerra, S. - J. Org. Chem., 61, 6849 (1996).

69. Helgaker, T.; Jaszunski, M.; Ruud, K. Chem. Rev. 1999, 99, 293.

70. Casanovas, J.; Alemán, C. - J. Mat. Sci., 37, 3589 (2002).

71. Triíto, I.; Marestin, C.; Boggioni, L.; Zetta, L.; Provasolli, A.; Ferro, D. R. - Macromolecules, 33, 8931 (2000).

72. Carbone, P.; Ragazzi, M. Triíto, I.; Boggioni, L.; Ferro, D. R. - Macromolecules, 36, 891 (2003).

73. Alemán, C.; Muñoz-Guerra, S. - J. Polym. Sci. Part B: Polym. Phys., 34, 963 (1996).

74. Kofranek, M.; Kóvar, T.; Linscha, H.; Karpfen, A. - J. Mol. Struct. (Theochem), 259, 181 (1992).

75. Rabias, I.; Howlen, B. J. - Comput. Theor. Polym. Sci., 11, 241 (2001),

76. Panchenko, Y. N. - J. Mol. Struct. (Theochem), 567, 217 (2001).

77. Alemán, C.; Domingo, V. M.; Fajaría, Ll.; Karpfen, A. J. Org. Chem., 63, 1041 (1998).

78. O'Malley, P. J. - J. Comput. Chem., 20, 1292 (1999).

79. Arizzi, S.; Mott, P. H.; Suter, U. W. - J. Polym. Sci. Part B: Polym. Phys., 30, 415 (1992).

80. Curcó, D.; Zanuy, D.; Alemán, C. - J. Comput. Chem., 24, 1208 (2003).

81. Gusev, A. A.; Müller-Plathe, F.; van Gunsteren, W. F.; Suter, U. W. - Adv. Polym. Sci., 116, 207 (1994).

82. Hofmann, D.; Fritz, L.; Ulbrich, J.; Schepers, C.; Böhning, M. - Macromol. Theory Simul., 9, 233 (2000).

83. Muller-Plate, F. - J. Chem. Phys., 103, 4346 (1995). 
84. Zanuy, D.; Alemán, C.; Muñoz-Guerra, S. - J. Polym. Sci. Part B: Polym. Phys., 40, 2037 (2002).

85. Mogri, Z.; Paul, D. R. Polymer, 42, 2531 (2001).

86. Zanuy, D.; Alemán, C.; López-Carrasquero, F.; Báez, M. E.; García-Álvarez, M.; Laso, M.; Muñoz-Guerra, S. Macromol. Chem. Phys., 202, 564 (2001).

87. Zanuy, D.; León, S.; Alemán, C.; Muñoz-Guerra, S. Polymer, 41, 4169 (2000).

88. León, S.; Zanuy, D.; Alemán, C. - J. Comput. Chem., 23, 685 (2002).

89. Zanuy, D.; Zazueta, A. D.; Alemán, C. - Polymer, aceptado para publicación (2003).

90. Grubb, D. T. - En Materials Science and Technology, Vol. 12; Cahn, R. W.; Haasen, P.; Kramer, E. J. (Eds.), pp: 301-356 (1993).
91. Nicholson, T. M.; Davies, G. R.; Ward, I. - Polymer, 35, 4259 (1994).

92. Tashiro, T. M.; Kobasashi, M.; Tadokoro, N. Macromolecules, 10, 413 (1977).

93. Rutledge, G. C. - Macromolecules, 30, 2785 (1997).

94. Thislehwaite, T.; Jakeways, R.; Ward, I. M. - Polymer, 29, 61 (1988).

95. Hine, P. J.; Ward, I. M. - J. Mat. Sci., 31, 371 (1996).

96. Fakirov, S.; Eustatiev, M. - Polymer, 31, 431 (1990).

97. Auriemma, F.; Corradini, P.; De Rosa, C.; Guerra, G.; Petraccone, V.; Bianchi, R.; Di Dino, G. Macromolecules, 25, 2490 (1992).

98. Kremer, K. - Macromol. Chem. Phys., 204, 181 (2003).

Recebido: 27/03/03

Aprovado: 09/09/03 